\title{
Les mycotoxines : quelles réponses de la recherche à cette problématique
}

\author{
Nadine Zakhia-Rozis ${ }^{1}$ \\ Sabine Schorr-Galindo ${ }^{2}$ \\ ${ }^{1}$ Cirad \\ Département «Performances des Systèmes \\ de Production et de Transformation \\ Tropicaux » (Persyst) \\ TA B-DIR/09 \\ Avenue Agropolis \\ 34398 Montpellier cedex 5 \\ France \\ <nadine.zakhia-rozis@cirad.fr> \\ ${ }^{2}$ Université de Montpellier 2 \\ UMR Qualisud \\ cc 023 \\ Place Eugène Bataillon \\ 34095 Montpellier cedex 05 \\ France \\ <Sabine.Galindo@univ-montp2.fr>
}

Ce numéro thématique est consacré à une question qui n'est pas nouvelle puisque dès les années 1960, la contamination des arachides africaines par l'aflatoxine et ses conséquences en termes de santé humaine et animale avaient déjà donné lieu à des décisions européennes contraignantes pour la filière et les échanges Nord-Sud.

Depuis cinquante ans, la présence de mycotoxines diverses a été révélée dans nombre de produits végétaux (céréales, café, cacao, fruits) et animaux (lait, abats), ce qui en fait réellement une question latente de santé publique.

Mais d'autres questions relatives à la sûreté sanitaire des aliments comme les résidus de pesticides, les métaux lourds, le parabène, la dioxine, etc. ont été bien plus médiatisées...

Néanmoins, les consommateurs sont de plus en plus sensibilisés à la qualité sanitaire de leurs aliments et à la manière dont ils sont produits, transformés, commercialisés. Les crises récentes liées à la présence de prions, de bactéries (Escherichia coli, Listeria monocytogenes, Salmonella spp, etc.) ont marqué les esprits.

Le dossier que nous présentons dans ce numéro thématique n'est pas brûlant, mais....

Les mycotoxines sont des métabolites secondaires produits par certains types de moisissures (ou champignons filamenteux) telles que celles appartenant aux genres Aspergillus, Penicillium et Fusarium. L'ingestion de produits contaminés par les mycotoxines entraîne des problèmes de santé publique pour l'homme (diminution de l'immunité générale, apparition de cancers hépatiques, rénaux ou digestifs, neurotoxicité) ainsi qu'une diminution de la productivité des animaux, et même leur mortalité. De plus, des pertes économiques significatives sont souvent associées à la contamination de denrées agroalimentaires par les mycotoxines, du fait du rejet des lots contaminés lors de transactions commerciales. Cela affecte tout particulièrement les exportations de produits d'origine tropicale du Sud, et notamment vers l'Union européenne, du fait du renforcement des normes et réglementations. Les productions européennes ne sont pas non plus épargnées face à certaines exigences de pays comme le Japon ou les États-Unis d'Amérique. Prévenir, maîtriser ou éliminer les mycotoxines constitue donc une priorité pour assurer la qualité sanitaire des aliments.

Certaines mycotoxines ont fait l'objet d'études et de recherches poussées afin de comprendre leurs mécanismes d'action sur la santé humaine et animale ainsi que leur voie de production par les moisissures et leur occurrence dans les denrées alimentaires. Ce sont principalement les aflatoxines, l'ochratoxine A, les trichothécènes dont le déoxynivalénol et les toxines T-2 et HT-2, les fumonisines et la zéaralènone. Les aflatoxines contaminent surtout les arachides, les fruits secs et les fruits à coques; l'ochratoxine A se retrouve dans le café, le cacao, le vin et dans les produits de la 
filière porcine (charcuterie, abats) (ces animaux ayant consommé des productions végétales contaminées); les trichothécènes, les fumonisines et la zéaralènone contaminent essentiellement les céréales.

Les réglementations concernant les teneurs maximales de mycotoxines autorisées dans les aliments se sont renforcées depuis une dizaine d'années, notamment dans l'Union européenne et aux États-Unis, alors qu'elles sont quasi inexistantes dans plusieurs pays du Sud qui sousestiment encore la gravité du problème. De même, les contrôles sanitaires obligatoires et systématiques dans les pays du Nord permettent de protéger les consommateurs, en leur évitant d'ingérer des aliments fortement contaminés par les mycotoxines. Cela n'est pas le cas pour les populations du Sud qui, du fait de l'absence de contrôles sanitaires et du rejet par les importateurs des lots contaminés, consomment fréquemment leurs produits locaux fortement contaminés. Pour faire respecter les normes, il faut des contrôles et pour cela, il faut disposer de méthodes d'analyse fiables, rapides et peu coûteuses et sensibles, les niveaux de contamination étant de l'ordre du microgramme par kilogramme. Les méthodes analytiques telles que les méthodes chromatographiques ou les méthodes immuno-enzymatiques permettent de détecter et de quantifier les mycotoxines dans les aliments. Plusieurs études inter-laboratoires ont permis de standardiser ces méthodes, en se basant sur des échantillons témoins ayant une teneur contrôlée en mycotoxines. L'échantillonnage et la préparation de l'échantillon avant analyse sont des étapes très importantes pour assurer la représentativité de l'analyse et elles sont aussi réglementées.

Contrairement à des idées assez communément admises il y a quelques années, la contamination n'est pas seulement liée à de mauvaises conditions de stockage des matières premières agricoles. La contamination par les mycotoxines peut débuter dans le champ, en fonction des pratiques culturales et des conditions climatiques et des maladies que peuvent développer les plantes, certaines moisissures toxinogènes étant phytopathogènes ou profitant d'un état altéré pour se développer. Elle peut également intervenir lors des opérations de post-récolte (stockage, séchage, transport) dans le cas d'infrastructures insuffisantes. La décontamination des produits finis n'est possible que par des traitements chimiques souvent agressifs, non autorisés pour l'alimentation humaine et qui altèrent la qualité des produits. Il serait également très coûteux de diminuer la contamination par les mycotoxines uniquement à la fin de la filière.

Il est donc essentiel de prévenir la contamination plutôt que d'essayer de la réduire. Pour cela, une approche intégrée et systématique, inspirée de la démarche "Analyse des dangers et des points critiques " (HACCP), a été appliquée dans différentes filières agricoles et alimentaires, afin d'identifier les sources potentielles d'apparition des moisissures toxinogènes et des mycotoxines, aux différentes étapes de la filière. Cela permet d'anticiper la contamination et d'apporter des solutions curatives ou préventives quand nécessaire, avant le stade du produit fini.

Progressivement, les recherches se sont orientées vers de nouvelles méthodes et démarches permettant de prévenir et maîtriser le problème que pose la contamination par les mycotoxines. Il s'agit de la sélection de variétés de plantes résistantes aux moisissures toxinogènes, de la lutte biologique par l'action de microorganismes bénins pouvant concurrencer le développement des moisissures toxinogènes dans le champ, ainsi que de l'identification de biomarqueurs permettant de tracer la contamination par les mycotoxines (dans le sang humain par exemple) et de la diminuer en intervenant sur l'alimentation.

Ce numéro thématique aborde la problématique des mycotoxines en présentant quelques filières (céréales, arachide, café, pisciculture) pouvant pâtir de ces contaminants. Il analyse aussi l'effet des pratiques culturales et des conditions agro-édapho-climatiques sur le développement des moisissures responsables de la production des mycotoxines ainsi que l'impact des traitements post-récolte. Il fait également le point sur les techniques analytiques permettant de déterminer les teneurs de ces contaminants dans les produits alimentaires, certaines pouvant servir d'outils d'aide à la décision par rapport à ce risque sanitaire et économique. 